\title{
Analgesia obstétrica utilizando a técnica de bloqueio paravertebral torácico bilateral: Relato de experiência em uma maternidade de risco habitual
}

\author{
Obstetric analgesia using a bilateral thoracic paravertebral block technique: An experience report \\ in a usual risk maternity
}

Analgesia obstétrica mediante técnica de bloqueo paravertebral torácico bilateral: Relato de una experiencia en maternidad de riesgo habitual

\author{
Adalgiso Feijó Malaguez \\ ORCID: https://orcid.org/0000-0001-6556-1691 \\ Universidade Franciscana, Brasil \\ E-mail: amalaguez@ufn.edu.br \\ Rosiane Filipin Rangel \\ ORCID: https://orcid.org/0000-0003-4059-4176 \\ Universidade Franciscana, Brasil \\ E-mail: rosiane@ufn.edu.br \\ Anna Luiza Frazzon Teixeira \\ ORCID: https://orcid.org/0000-0002-3638-3520 \\ Universidade Franciscana, Brasil \\ E-mail: anna_fteixeira@hotmail.com \\ Gabriela Maciel \\ ORCID: https://orcid.org/0000-0002-9776-5549 \\ Universidade Franciscana, Brasil \\ E-mail: gabimaciel.gm@ hotmail.com \\ Alexandre Antonio Naujorks \\ ORCID: https://orcid.org/0000-0001-6111-662X \\ Universidade Franciscana, Brasil \\ E-mail: alexandre.cardio@gmail.com
}

\begin{abstract}
Resumo
Objetivo: Relatar uma experiência de utilização do bloqueio paravertebral torácico como alternativa à analgesia obstétrica. Método: Relato de experiência sobre a realização de uma técnica de analgesia, que foi utilizada para contribuir com a analgesia obstétrica e o parto natural de uma gestante com indicação de cesárea por fobia à dor do parto natural. A vivência foi numa maternidade de um hospital filantrópico, de médio porte, na zona da campanha, no estado do Rio Grande do Sul. Resultados: O bloqueio paravertebral torácico bilateral foi o método analgésico escolhido por não utilizar o neuro-eixo como substrato anatômico e não produzir flutuação hemodinâmica na paciente e consequências no feto. A gestante após receber analgesia, não apresentou dor no primeiro estágio do trabalho de parto. Essa técnica, permitiu nesse caso que a indicação de cesárea por fobia à dor do parto fosse revista pela equipe obstétrica e o parto desenvolveu-se de maneira natural. A equipe de enfermagem da maternidade é parte central na utilização desta técnica, para conduzir a monitorização e os cuidados da parturiente. Conclusão: A analgesia obstétrica contribui para a humanização do parto natural. É preciso fomentar essa prática, já que por si só tende a aumentar o número de partos normais, haja vista, que na primeira consulta pré-natal, algumas gestantes têm o desejo de realizar parto cesáreo por medo da dor.
\end{abstract}

Palavras-chave: Parto normal; Analgesia obstétrica; Bloqueio epidural; Bloqueio paravertebral.

\begin{abstract}
Objective: To report an experience of using thoracic paravertebral block as an alternative to obstetric analgesia. Method: Experience report on the performance of an analgesia technique that was used to contribute to obstetric analgesia and natural childbirth of a pregnant woman with indication for cesarean section due to phobia of the pain of natural childbirth. The experience was in a maternity ward of a medium-sized philanthropic hospital, in the countryside, in the state of Rio Grande do Sul. Results: Bilateral paravertebral thoracic block was the analgesic method chosen for not using the neuro-axis as an anatomical substrate and do not produce hemodynamic fluctuation in the patient and consequences in the fetus. After receiving analgesia, the pregnant woman did not experience pain in the first stage of labor. This technique, in this case, allowed the indication of cesarean section due to phobia of labor pain to be reviewed by the obstetric team and the childbirth developed in a natural way. The maternity nursing team is central to the use of this technique, to conduct monitoring and care for the parturient. Conclusion: Obstetric analgesia
\end{abstract}


contributes to the humanization of natural childbirth. It is necessary to encourage this practice, since it alone tends to increase the number of natural births, considering that in the first prenatal consultation, some pregnant women have the desire to perform cesarean delivery for fear of pain.

Keywords: Natural childbirth; Analgesia, Obstetrical; Epidural block; Paravertebral block.

\section{Resumen}

Objetivo: Informar una experiencia de uso del bloqueo paravertebral torácico como alternativa a la analgesia obstétrica. Método: Informe de experiencia sobre la realización de una técnica de analgesia que sirvió para contribuir a la analgesia obstétrica y parto natural de una gestante con indicación de cesárea por fobia al dolor del parto natural. La experiencia fue en una maternidad de un hospital filantrópico de tamaño mediano, en el campo, en el estado de Rio Grande do Sul. Resultados: El bloqueo torácico paravertebral bilateral fue el método analgésico elegido por no utilizar el neuroeje como sustrato anatómico y no producen fluctuación hemodinámica en el paciente y consecuencias en el feto. Después de recibir analgesia, la embarazada no experimentó dolor en la primera etapa del parto. Esta técnica, en este caso, permitió que la indicación de cesárea por fobia al dolor del parto fuera revisada por el equipo de obstetricia y el parto se desarrollara de forma natural. El equipo de enfermería de maternidad es fundamental para el uso de esta técnica, para realizar el seguimiento y el cuidado de la parturienta. Conclusión: La analgesia obstétrica contribuye a la humanización del parto natural, es necesario incentivar esta práctica, ya que por sí sola tiende a incrementar el número de partos naturales, considerando que en la primera consulta prenatal, algunas gestantes tienen el deseo de realizar cesárea. por miedo al dolor.

Palabras clave: Parto natural; Analgesia obstétrica; Bloqueo epidural; Bloqueo paravertebral.

\section{Introdução}

Receber atenção durante o trabalho de parto que ofereça a possibilidade de controle da dor quando, e, se necessário, é um direito da mulher. No entanto, sabe-se que, por vezes, esses direitos as gestantes não são contemplados, já que os dados apontam para uma oferta de analgesia obstétrica aquém das necessidades. No Brasil cerca de $30 \%$ das parturientes recebem analgesia obstétrica, no entanto quando o olhar é para as técnicas farmacológicas esses números não passam de 3,5\% (Brasil, 2006). Na comparação com países desenvolvidos da Europa e dos Estados Unidos, a analgesia obstétrica farmacológica atinge $60 \%$ (Penuela et al, 2019), números em torno de 17 vezes maior que no contexto nacional.

Outro dado que deve ser considerado é que o Brasil, mundialmente, é um dos países com as maiores taxas de cesarianas, em torno de $56 \%$ (Batista Filho \& Rissin, 2018). Nessa direção, ao analisar que 28\% das gestantes manifestam na primeira consulta pré-natal o desejo de terem seus partos por via cirúrgica, devido ao medo da dor (Fiocruz, 2014), a falta de analgesia obstétrica que se evidencia, é um dos fatores que podem contribuir com essas estatísticas.

Frente a isso, compreende-se que a analgesia obstétrica é um fator que precisa ser discutido e implementado na assistência ao parto, com vistas a contribuir na humanização do parto, bem como na redução dos índices de cesárea (Maia, 2010, Novo et al, 2017). Cabe salientar que, o trabalho de parto tem quatro fases, quais sejam: primeira - dilatação; segunda expulsão; terceira - secundamento e quarta - pós-parto. A partir do mecanismo fisiológico de cada período, a dor gerada durante o parto chega ao Sistema Nervoso Central (SNC) através das vias espino-talâmicas em raízes posteriores de T10, T11, T12 e L1 no primeiro estágio e, S2, S3 e S4 no segundo e terceiro estágios (Brasil, 2017).

Nessa direção, as opções para manejo não farmacológico da dor podem auxiliar a parturiente no alívio da dor e devem ser sempre a primeira escolha (Brasil, 2017). Dentre essas, destaca-se: apoio contínuo da gestante durante o trabalho de parto (como o acompanhamento de doulas), imersão em água ou banho terapêutico, mobilidade e alternância de posições, técnicas de relaxamento, acupuntura, aromaterapia e massagem. Esses métodos baseiam-se em conhecimentos estruturados, mas que não necessitam de equipamentos sofisticados, podendo ser aplicados, até mesmo, pelo acompanhante de escolha da gestante (Gayeski \& Brüggemann, 2010; Silva, Cunha \& Araújo, 2020).

No que tange a analgesia obstétrica farmacológica, essa pode ser realizada pela administração de drogas endovenosas, normalmente da classe dos opioides, que produzem efeito analgésico ao se ligarem a receptores endorfínicos (Mu, Kappa e Delta). Esta ligação permite a formação de corrente de potássio para fora do neurônio, causando hiperpolarização no neurônio 
de segunda ordem, localizado no corno posterior da medula espinhal e assim dificultando o estabelecimento do sinal elétrico doloroso em direção ao SNC (Morgan \& Mikhail, 2018). Essa técnica tem seu uso limitado por seus efeitos depressores do SNC, tanto na mãe quanto no feto (Anim-Somuah, Smyth \& Jones, 2011).

Outra forma de produzir analgesia obstétrica farmacológica é a abordagem de neuro-eixo. Nestas técnicas, os anestésicos locais são injetados próximo às raízes específicas que conduzem potencial de ação elétrico por meio das vias de dor (Bloqueio Epidural, Subaracnoídeo ou ambos). Os anestésicos locais bloqueiam canais de Sódio em vias dolorosas e evitam que haja formação deste potencial de ação elétrico, bloqueado a sensação de dor (Chau \& Tsen, 2018). Ressalta-se que as informações/orientações acerca das medidas farmacológicas e não farmacológicas devem fazer parte das consultas de prénatal, visando esclarecer para a gestante as possibilidades, riscos e benefícios da utilização dessas no trabalho de parto (Silva et al, 2020).

A partir do exposto, pretende-se com esse relato expandir as possibilidades técnicas de uso de analgesia farmacológica, ao descrever a injeção de anestésico local no espaço paravertebral torácico bilateralmente (Okutomi et al, 2002; Antipin et al, 2014), para uso em analgesia obstétrica farmacológica. Assim, objetiva-se com o estudo, relatar uma experiência de utilização do bloqueio paravertebral torácico como alternativa à analgesia obstétrica que aborda neuro-eixo.

\section{Metodologia}

Trata- se de um estudo descritivo, do tipo relato de experiência, de abordagem qualitativa. Relata-se a realização da execução do bloqueio paravertebral torácico bilateral para prover analgesia obstétrica. Para tanto, foi selecionada uma gestante de 29 anos, com dois partos vaginais prévios, 40 semanas de gestação e que estava em trabalho de parto. A escolha se deu ao fato de a parturiente ter fobia à dor do parto. O local do estudo foi uma maternidade de risco habitual, de um hospital filantrópico, de médio porte, localizado na Zona da campanha, no estado do Rio Grande do Sul - RS. Cabe salientar que no referido hospital, há apenas um anestesiologista de plantão, responsável pelas cirurgias obstétricas e de emergência advindas do pronto atendimento e de pacientes internados. A vivência foi realizada no mês de abril de 2020, após consentimento da parturiente e delineamentos acordados entre profissionais da equipe multiprofissional. Este estudo foi aprovado pelo Comitê de Ética em Pesquisa da Universidade Franciscana, parecer nº 4.442.526.

\section{Resultados e Discussão}

Apresenta-se a experiência em dois eixos para melhor visualização e compreensão: Anatomia do espaço paravertebral torácico - em que é discutido acerca da técnica considerando a anatomia humana; Relatando a Experiência - em que é descrito o caso de uma gestante, bem como o desfecho do parto a partir da utilização de analgesia obstétrica com a técnica de bloqueio paravertebral torácico bilateral.

\subsection{Anatomia do Espaço Paravertebral Torácico}

Trata-se de um espaço em forma de cunha delimitado anterolateralmente pela pleura parietal, medialmente pela coluna vertebral, e posteriormente pelo processo transverso vertebral e pelo ligamento costotransverso. Os nervos intercostais com seu ramo dorsal, os ramos comunicantes e a cadeia simpática ao longo da coluna são os conteúdos de tecido nervoso do espaço paravertebral torácico (Figura 1). O potencial de ação nestes nervos será bloqueado quando se injeta anestésico local neste espaço (Bouman et al, 2017). 
Figura 1. Espaço paraverterbral torácico, vista tridimensional.

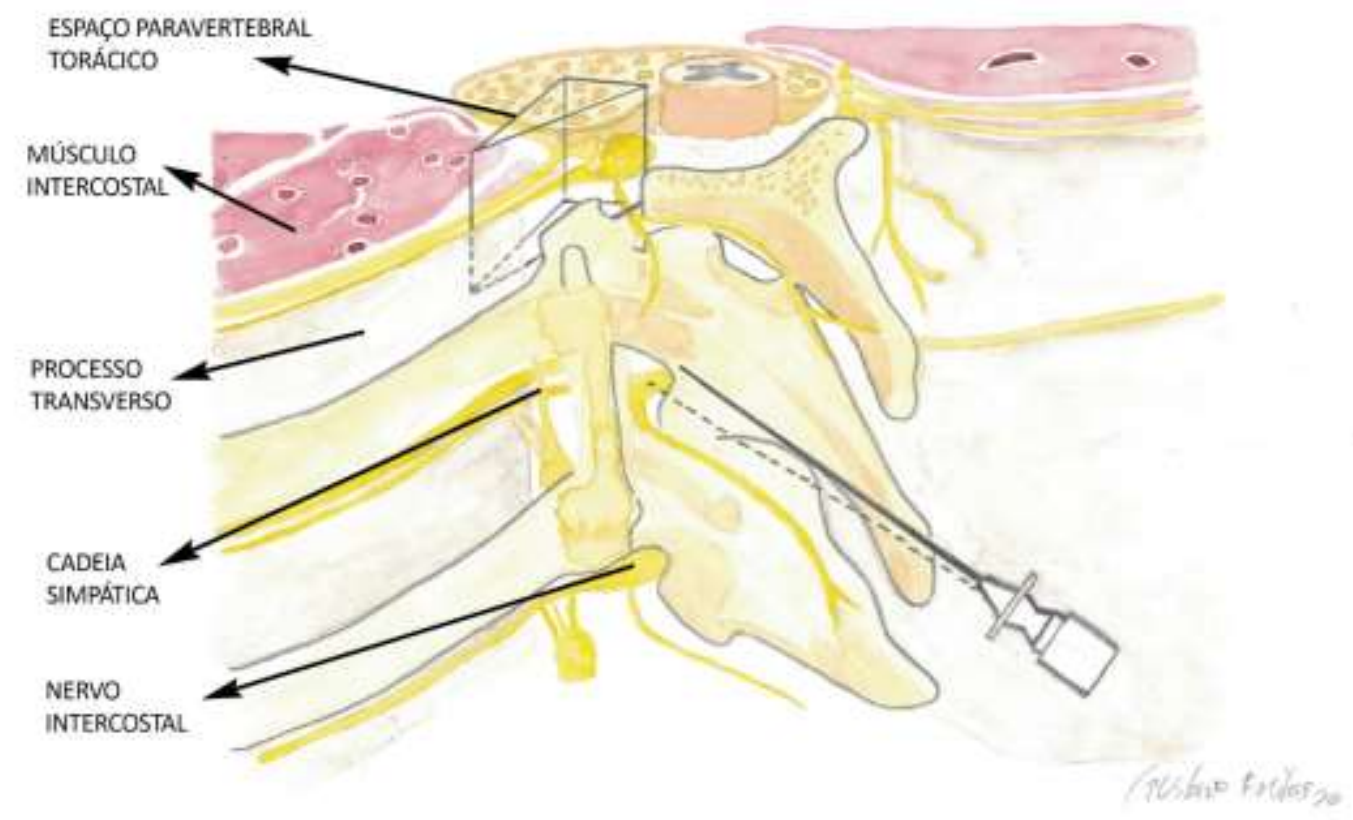

Fonte: Adaptado de Dodd; Hunsley (2011).

A utilização do bloqueio paravertebral torácico bilateral tem facilidade da realização da punção em comparação com a punção epidural, menor custo, poucos efeitos colaterais, a não necessidade de monitorização hemodinâmica contínua (Antipin et al, 2014). Compreende-se que esses fatores podem fazer desta técnica uma grande aliada na prática do parto humanizado.

\subsection{Relatando a Experiência}

Gestante de 29 anos, tercigesta, com dois partos vaginais prévios, foi internada com 40 semanas de gestação, em trabalho de parto, primeiro estágio, bolsa íntegra, em um hospital filantrópico de referência, na zona da campanha no estado do RS. Essa tem uma população de 33.624 habitantes. No referido local, existe centro materno-infantil para gestantes de risco habitual, onde há um trabalho multiprofissional, em que as intervenções obstétricas são acordadas e discutidas entre os diferentes profissionais, visando a humanização do parto. A maternidade consta de nove leitos para o Sistema Único de Saúde (SUS), além de duas salas para parto normal, uma sala de preparo e uma sala de exame para pacientes extra-hospitalares. Alguns métodos não farmacológicos são oferecidos, como: bola suíça, banhos mornos, e suporte de acompanhantes durante o parto. Possui um regime de plantões à distância para médicos nas áreas de pediatria, obstetrícia, cirurgia geral, clínica médica e anestesiologia e, um anestesiologista de plantão por dia.

Não raro, apresentam-se concomitantemente gestantes em trabalho de parto e outras parturientes internadas, com possibilidade de indicação de cesariana. Esta é uma das razões que torna o serviço, frequentemente, incapaz de oferecer analgesia obstétrica. No dia da vivência, nesta maternidade havia outra gestante com indicação de parto cesáreo por iteratividade (Montenegro, 2012).

Ao exame obstétrico, paciente apresentava-se no momento da internação em primeiro estágio do trabalho de parto, fase passiva, com quatro centímetros de dilatação. Em uma hora, evolui para primeiro estágio de parto, fase ativa, com três contrações em dez minutos, com duração de 40 segundos cada e batimentos cardíacos fetais de 136 por minuto. Paciente relatava dor e sofrimento na última gestação. Existia, por parte do obstetra, uma possibilidade a indicar cesariana, devido à 
resistência da paciente em prosseguir o trabalho de parto, devido ao medo de sentir dor, sendo que a mesma pediu para que a cesariana fosse indicada.

Após discussão com obstetra, pediatra e enfermeira, optou-se pela tentativa de manutenção do trabalho de parto com analgesia obstétrica (devido à fobia à dor do parto da parturiente), com intuito de não converter o parto normal à cesariana por exclusiva razão de dor da paciente. Entende-se que o medo à dor é um dos fatores que contribui com o aumento nos índices de cesarianas no Brasil (Fernandes et al, 2017).

Conversou-se com a gestante explicando a possibilidade farmacológica para redução da dor, isso à levou a solicitar analgesia obstétrica, procedendo-se na sequência a realização da mesma. A técnica de escolha foi o bloqueio paravertebral torácico bilateral, que não produz variações hemodinâmicas (Antipin et al, 2014) e permite que a analgesia seja assistida pela equipe de enfermagem, caso o anestesiologista precise se ausentar da sala operatória. Essa ausência é devido as características do Hospital, onde o anestesiologista frequentemente pode ser demandado a suprir procedimentos cirúrgicos e, essa falta de assistência anestesiológica, inviabiliza a técnica de analgesia obstétrica utilizando o bloqueio de neuro-eixo tradicional (peridural), em razão da produção de flutuações hemodinâmicas inerentes dessa técnica (Anim-Somuah, Smyth \& Jones, 2011).

Pela Escala Visual Analógica da dor, a paciente relacionou a dor máxima (10/10) quando em contração. Foi monitorizada, com monitor multiparâmetro: Pressão Arterial (PA) de 110/75 mmHg; Frequência Cardíaca (FC) de 100 bpm e saturação de $\mathrm{O} 2$ de $98 \%$ em ar ambiente. O bloqueio paravertebral torácico foi realizado em nível de décima vértebra torácica. Com a agulha espinhal tipo Quinke $22 \mathrm{G}$, penetrou-se em direção ao processo transverso e a agulha tocou o osso da vértebra; recuou-se dois milímetros e desviou-se a agulha em direção cefálica, "fugindo" do processo transverso e passando o ligamento costotranverso, sentindo um "clique". Foram injetados $15 \mathrm{ml}$ de ropivacaína $0,2 \%$ em cada lado.

Após quinze minutos do bloqueio a paciente relacionou alívio total da dor, deambulou, fez manobras na bola suíça e relatava sensação de puxo. Padrão hemodinâmico manteve-se estável (menos de $20 \%$ de variação). Após uma hora, paciente entrou na fase expulsiva do trabalho de parto e em cinco minutos houve nascimento, sem laceração do períneo. Não houve episiotomia, e paciente relatou dor forte no momento da expulsão. Recém-nascido teve os seguintes parâmetros: Apgar:8/10, FC: 130 batimentos por minuto, Saturação de O2 de $98 \%$ em ar ambiente.

O bloqueio paravertebral torácico bilateral é uma alternativa que tem o intuito de contribuir como técnica de analgesia obstétrica farmacológica devido: 1) menor complexidade, comparado a outras técnicas; 2) menor risco de complicações; 3 ) possibilidade de realização em pacientes com distúrbios de coagulação sanguínea ou uso de anticoagulantes; 4) menor custo (Okutomi et al, 2002; Antipin et al, 2014).

Ressalta-se o papel da equipe multidisciplinar na execução da técnica, pois necessita de entendimento do benefício produzido entre os agentes promotores do parto humanizado, e principalmente, um sincronismo entre a enfermeira da maternidade e o médico anestesiologista para executar a analgesia com sucesso em termos de assistência no período periparto. Salienta-se que a interação da equipe multiprofissional, preconizada pela Organização Mundial de Saúde (OMS) e Ministério da Saúde (MS), contribui significativamente para que o trabalho de parto, parto e nascimento sejam uma experiência agradável (Oliveira et al, 2016).

Em suma, por ser uma técnica incipiente, é necessário que as impressões, sejam confirmadas com outros estudos multicêntricos, comparando o bloqueio paravertebral torácico bilateral com outras técnicas de analgesia obstétrica (abordagem de neuro-eixo).

\section{Considerações Finais}

Considera-se que a analgesia obstétrica contribui para a humanização do parto natural, sendo que é direito da mulher 
brasileira, reconhecido por lei, quando assim desejar, recebê-la. É preciso fomentar essa prática, já que por si só tende a aumentar o número de partos naturais, haja visto, que na primeira consulta pré-natal, algumas gestantes manifestam a vontade de realizar parto cesáreo por medo da dor. Entende-se que ao oferecer alternativas para aplacar a dor, reduzirão essas manifestações. Em suma, o bloqueio paravertebral torácico bilateral, por ser uma técnica incipiente, necessita de mais estudos para que as impressões apresentadas nesse relato sejam confirmadas

\section{Referências}

Anim-Somuah, M., Smyth, R. M., \& Jones, L. (2011). Epidural versus non-epidural or no analgesia in labour. The Cochrane database of systematic reviews, (12), CD000331. https://doi.org/10.1002/14651858.CD000331.pub3

Antipin, É. É., Uvarov, D. N., Nedashkovskiı̌, É. V., \& Kushev, I. P. (2014). Epidural analgesia in the first stage of labor--is there an alternative? Anesteziologiia i reanimatologiia, (1), 18-22.

Batista Filho, M., \& Rissin, A. (2018). A OMS e a epidemia de cesarianas. Revista Brasileira de Saúde Materno Infantil, 18(1),34. https://doi.org/10.1590/1806-93042018000100001

Bouman, E., Sieben, J. M., Balthasar, A., Joosten, E. A., Gramke, H. F., van Kleef, M., \& Lataster, A. (2017). Boundaries of the thoracic paravertebral space: potential risks and benefits of the thoracic paravertebral block from an anatomical perspective. Surgical and radiologic anatomy: SRA, 39(10), 1117-1125. https://doi.org/10.1007/s00276-017-1857-4

Chau, A., \& Tsen, L. C. (2018). Update on Modalities and Techniques for Labor Epidural Analgesia and Anesthesia. Advances in anesthesia, 36(1), 139-162. https://doi.org/10.1016/j.aan.2018.07.006

Fiocruz. (2014) Nascer no Brasil - Inquérito Nacional sobre Parto e Nascimento. http://www6.ensp.fiocruz.br/nascerbrasil/.

Gayeski, M. E., \& Brüggemann, O. M. (2010). Métodos não farmacológicos para alívio da dor no trabalho de parto: uma revisão sistemática. Texto \& Contexto - Enfermagem, 19(4), 774-782. https://doi.org/10.1590/S0104-07072010000400022

Maia, M. B. (2010). Humanização do parto: política pública, comportamento organizacional e ethos profissional: Editora Fiocruz.

Ministério da Saúde. (2017) Diretrizes Nacionais de Assistência ao Parto Normal. Brasília - DF.

Ministério da Saúde. Pesquisa Nacional de Demografia e Saúde da Criança e da Mulher - PNDS (2006) dimensões do processo reprodutivo e da saúde da criança/ Ministério da Saúde, Centro Brasileiro de Análise e Planejamento. - Brasília: Ministério da Saúde.

Morgan, G. E., \& Mikhail, M.S., (2018). Clinical Anesthesiology. (6 ${ }^{\mathrm{a}}$ ed.) LANGE.

Okutomi, T., Taguchi, M., Amano, K., \& Hoka, S. (2002). Paravertebral block for labor analgesia in a parturient with idiopathic thrombocytopenia.Masui. The Japanese journal of anesthesiology, 51(10), 1123-1126.

Penuela, I., Isasi-nebreda, P., Almeida, H., López, M., Gomez-Sanchez, E., \& Tamayo, E. (2019). Epidural analgesia and its implications in the maternal health in a low parity comunity. BMC Pregnancy Childbirth, 19(1):52. https://doi.org/10.1186/s12884-019-2191-0

Dodd, M., \& Hunsley, J. (2011). Thoracic paravertebral block: landmark techniques anaesthesia tutorial of the week 224 . https://resources.wfsahq.org/atotw/thoracic-paravertebral-block-landmark-techniques/

Silva, A. D. V., Cunha, E. A., \& Araújo, R. V. (2020). The benefits of integrative and complementary practices in childbirth work. Research, Society and Development, 9(7): 1-16, e614974468. http://dx.doi.org/10.33448/rsd-v9i7.4468

Montenegro, C. A. B. (2012). Operação cesária. In: Montenegro, C. A. B., Rezende Filho, J. Rezende obstetrícia fundamental. (12ª ed.): Guanabara Koogan; p. 634-46.

Novo, J., Pellicciari, C., Camargo, L., Bálsamo, S., \& Novo, N. (2017). Indicações de partos cesáreos em hospitais de atendimento ao Sistema Único de Saúde: baixo e alto riscos. Revista da Faculdade de Ciências Médicas de Sorocaba, 19(2), 67-71. https://doi.org/10.23925/1984-4840.2017v19i2a4

Fernandes, R. L.V., Damasceno, A. K. C., Herculano, M. M. S., Martins, R. S. T, \& Oriá M. O. B. (2017). Analgesia obstétrica farmacológica: um estudo sobre os desfechos obstétricos e neonatais. Revista Rene. 18(5):687-94. 10.15253/2175-6783.2017000500017

Silva, Y. A. P., Araújo, F. G., Amorim, T., Francisca Martins, E., \& Felisbino-Mendes, M. S. (2020). Analgesia obstétrica no trabalho de parto e sua associação com desfechos neonatais. Revista Brasileira de Enfermagem, 73(2), e20180757. https://dx.doi.org/10.1590/0034-7167-2018-0757

Oliveira, J., Campos, T., Souza, F., Davim, R., \& Dantas, J. (2016). Percepção de enfermeiros obstetras na assistência à parturiente. Revista de Enfermagem UFPE on line. 10(10, 3868-3875. 10.5205/reuol.9667-87805-1-ED1010201619 